\title{
First Responders' Crystal Ball: How to Scry the Emergency from a Remote Vehicle
}

\author{
Marco Roccetti ${ }^{(1)}$, Mario Gerla ${ }^{(2)}$, Claudio E. Palazzi ${ }^{(1,2)}$, Stefano Ferretti ${ }^{(1)}$, Giovanni Pau $^{(2)}$ \\ ${ }^{1}$ Dipartimento di Scienze dell'Informazione, Università di Bologna, \\ Mura Anteo Zamboni 7, 40127 Bologna, Italia \\ \{roccetti, cpalazzi,sferrett\}@cs.unibo.it \\ ${ }^{2}$ Computer Science Department, University of California Los Angeles, \\ Boelter Hall, Los Angeles, CA, 90095, USA \\ \{gerla,gpau\}@cs.ucla.edu
}

\begin{abstract}
Successes and failures during rescue operations after hurricane Katrina and the Twin Towers attack demonstrated the importance of supporting first responders with adequate means to perform their operations in an effective and safe way. From a networking point of view, one of the main challenges is that of providing first responders with multimedia information about the emergency as soon as possible, even from a remote location. To this aim, we designed an inter-vehicular communication system able to quickly discover and transmit real time multimedia information from around a crisis area to approaching first responders' vehicles. As vehicular communications are highly variable in nature, we endowed our system with a transmission range estimator that is put to good use to reduce the number of hops that a video triggering message sent by a vehicle will experience to reach its destination. Experimental results demonstrate the efficacy of our scheme in reducing the message delivery time and the traffic generated.
\end{abstract}

\section{Introduction}

The horrific stories of violent anarchy in the postKatrina New Orleans and the hundreds of casualties among first responders in the Twin Towers attack have reached each of us through our TV sets. These further tragedies made those disasters even more unbearable and demonstrated, once more time, the importance of supporting first responders with adequate means to perform their operations in an effective and safe way. Reliable communications, situation awareness from remote sites, and team coordination have to be guaranteed especially in extraordinary circumstances such as natural or terrorist disasters. Failures are unacceptable as they are paid in hundreds, thousands of human lives even among first responders [1].

Accurate and timely information is essential to prepare operations and to take action in the best and fastest possible way. To this aim, the hundreds of cameras and other sensors that are present today all around us should be exploited to provide multimedia information to policemen, firefighters, paramedics, and other operators when an emergency occurs [2]. Assume that first responders driving to the emergency area could trigger a live video stream generated by a video camera present around the emergency site and watch it on a screen in their vehicle, just by indicating the geographical area. This application would be equivalent to letting first responders scrying the emergency area with a crystal ball. Anyone could imagine an infinite number of useful applications. For instance, paramedics in an ambulance could watch the scene of the accident even before arriving on site and proactively organize first aid operation; police vehicles could broaden their view by receiving videos from around a neighborhood; firefighters could have a comprehensive view of an urban fire while still driving to the area; specialized operators could study the best strategy to intervene in a contaminated area to minimize the danger; etc.

Obviously, transmissions related to such applications have to be feasible even in areas without communication infrastructure along the road or in case of a major disaster that had destroyed it. Therefore, inter-vehicular multi-hop wireless transmissions represent the best option to guarantee dependable communications between a video camera (or any other utilizable device) located in the emergency area and an approaching vehicle. Indeed, this kind of transmission 
will soon be possible thanks to the on-going development of the IEEE 802.11p/DSRC technology for vehicular communications [3].

In this context, a well known technical problem is that of sending a triggering message to start live video streaming (or any other command) from a remote location which represents the area-of-interest, in a very fast way [4]. Depending on the distance between the requesting vehicle and the corresponding area-ofinterest, the triggering message can be sent either directly or through multiple hops; the latter obviously represents a more challenging case. Since we are considering a real-time application such as live video streaming, it is crucial that the triggering message reaches the area-of-interest very quickly. Several works in the field of drive safety indicate that the main problems impeding a fast message delivery in intervehicular communications is represented by a nonoptimal (i.e., too high) number of hops experienced by the message to cover its path and, more in general, an excessive number of transmissions [5, 6].

However, having a triggering message covering as fast as possible the path from its source to its destined area-of-interest represents a difficult task [4]. The problem is exacerbated by the fact that vehicles move very quickly, thus experiencing frequent changes in their networking conditions and requiring dynamically adaptable algorithms.

We hence focus on the challenging problem of how to send a triggering message to a remote area-ofinterest, through a multi-hop wireless connection among vehicles, which may hence experience highly variable transmission conditions. To this aim, we discuss a novel video triggering algorithm, named Fast Triggering, that is specifically designed for intervehicular communications. Fast Triggering is based on a distributed mechanism for the estimation of the backward and frontward transmission ranges of vehicles. These continuously updated estimations are then used to reduce the amount of hops that a triggering message has to experience in its path toward the destination.

For the sake of conciseness, in this paper we focus on the triggering of a live video streaming application. However, expressed considerations can be easily extended to any kind of data stream (e.g., sound, sensors' outcomes).

The rest of the paper is organized as follows. Section 2 places assumptions of the setting we are considering. Section 3 surveys algorithms proposed for fast multi-hop delivery of messages in a inter-vehicular communication context. The Fast Triggering algorithm is discussed in Section 4. Section 5 details the simulative environment we have adopted to evaluate the performance of schemes for forwarding video triggering messages. Experimental results are reported in Section 6. Finally, in Section 7, a conclusion is drawn.

\section{Vehicular communications scenario}

We consider a strip-shaped portion of road where vehicles move along, passing by very different surroundings (i.e., buildings, hills, curves). In this setting, vehicles travel at high speed and with potentially high density, thus being exposed to high variation in terms of transmission range and available bandwidth. It is crucial that protocols and applications be designed able to adapt to different transmission conditions.

For the sake of simplicity, vehicles are assumed to be endowed with about the same computational and transmission capabilities; our mechanism can be easily extended to work even in the case where these assumptions do not hold. Each vehicle is able to communicate with other vehicles in a P2P fashion even exploiting multiple hops. To this aim, the IEEE 802.11p/DSRC technology has been declared able to guarantee a maximum range of $1000 \mathrm{~m}$ under optimal conditions, or a smaller range at very high speeds (around $300 \mathrm{~m}$ for a car traveling at $200 \mathrm{Km} / \mathrm{h}$ ) [3]. Cameras may be located along the street-side or even on vehicles. Finally, a GPS device that provides accurate information about its position and trajectory is present on-board.

\section{Inter-vehicular transmissions: related work}

Sending a message to trigger a live video stream basically consists in the fast delivery, even through multi-hops, of a broadcast message from a vehicle to a certain area-of-interest. To forward the message toward its destination, geographical coordinates and store-carry-and-forward techniques can be exploited [4]. Many of these schemes follow the greedy principle, also exploited by geo-routing schemes, by which the message delivery will be faster if performed through longer leaps that bring it closer to its target. On a freeway this means that hops should be as long/few as possible to minimize the number of transmitted messages [5, 6].

To this aim, [7] proposes a backoff mechanism that 
reduces the frequency of message retransmissions when congestion is causing collisions. In [8], as soon as a car receives a message from a following vehicle along a strip, it refrains from forwarding it as the reception of this message is a clear confirmation that subsequent cars have already received it. Unfortunately, both these two schemes do not consider a very important factor in determining the final propagation delay of a message: the number of hops a message traverses before covering its whole area-ofinterest.

In [6], hops' minimization is achieved by individuating the farthest car within the source's backward transmission range, which has to forward the message. To this aim, jamming signals are emitted by each car with a duration that is directly proportional to the distance between the considered car and the message's source. The car with the longest jamming signal is clearly the farthest car from the source. Even if this guarantees a minimum number of hops to reach the destination, the time wasted to determine the next forwarder through jamming signals could make this scheme not suitable for a real-time scenario.

Instead, inspired by an algorithm that exploits a direct proportion between the distance from the message's sender and the priority of the considered vehicle in becoming the next forwarder [5], we propose a novel distributed algorithm that allows vehicles to estimate their transmission range and to exploit this value to reduce the number of forwarding hops.

\section{How to quickly trigger an emergency video}

Fast Triggering is designed to send video triggering messages, as fast as possible, to a certain area. To this aim, our algorithm steps through two different phases. First, during the estimation phase, vehicles exchange (few) hello messages to collect information in order to estimate their own transmission range. Specifically, they try to estimate the maximum distance, both frontward and backward, at which they would be heard by other vehicles in case they tried to send a message. Then, in the triggering phase, this estimation is put to good use to reduce the number of hops that a video triggering message will experience in its trip to destination.

The rationale of this scheme is that by including a continuously updated transmission range estimation in the triggering message, we let following cars to be aware of their position within the transmission range and have the farthest of them to forward the triggering message.

\subsection{Estimating the transmission range, efficiently}

To estimate its transmission range, each vehicle periodically tries to send a hello message and collects information included in hello messages sent by other vehicles. Time is divided into rounds and, at a certain random time chosen within each round, each vehicle tries to send a hello message. The first hello message sent in a certain round stops the sending procedure of other vehicles hearing that message. Therefore, in a certain area as large as the transmission range, only one hello message is sent per each round. Moreover, the hello messages mechanism is deadlock free as the MAC layer guarantees collision avoidance.

In each of these messages, the sender includes i) its own position, ii) its backward maximum distance (BMD) parameter, and iii) its frontward maximum distance (FMD) parameter. These two latter parameters represent the maximum distance from which another vehicle, backward or frontward respectively, has been heard by the considered one. Data utilized to determine these parameters are kept by each vehicle only for a certain amount of time, after which they are considered obsolete for the transmission range estimation.

Vehicles exploit hello messages to estimate the transmission range, i.e., estimated transmission range (ETR), both backward and frontward. To compute ETRs, both the highest distance from which another vehicle has been heard sending a hello message and the highest maximum distance advertised by heard hello messages are employed. Specifically, the backward ETR is obtained by considering only hello messages coming from following vehicles and is computed as the largest among all their included FMD values and all the distances from the vehicles that have generated them. Instead, the frontward ETR utilizes only hello messages sent by preceding vehicles and is equal to the largest value among all the advertised BMDs and the distances from the vehicles that have sent the considered hello messages.

Basically, each car can be both a sender and a receiver of hello (and triggering) messages. If we consider for simplicity only the case where triggering messages are always sent frontward (the backward case is just specular), then we have the following purpose for each message: 
i) Hello messages received from the back allow the receiver to compute the $\mathrm{BMD}$; this value will then be declared by the receiver in its hello messages as it were saying: "This BMD value is the farthest distance from which I have been able to hear another car behind me".

ii) Hello messages received from frontward includes the sender's BMD and position. They hence provide the receiver with information about the hearing capabilities of preceding cars. This is what the receiver needs in order to compute its frontward ETR that will be sent along with the triggering messages as it were saying: "This ETR value is the maximum frontward distance at which some car would be able to hear me”.

\subsection{Triggering a live video stream while driving}

When an operator on the vehicle (e.g., the ambulance) activates the remote video stream, a triggering message has to be sent in broadcast from that vehicle to the geographical area-of-interest very quickly (i.e., with a minimal number of hops). This is exactly what we try to achieve with the use of ETR. More in detail, the video triggering message contains information related to the video transmission that has to be activated but also the sender's position and its current ETR.

ETR represents how far a transmission is expected to go before the signal becomes to weak to be intelligible. Its value will be used by vehicles on the message's path to destination to determine which one among them will be the next forwarder of the triggering message. As the aim is that of minimizing the number of hops to reduce the propagation delay, the farthest possible vehicle from the sending one should be privileged to become the next forwarder. In particular, vehicles' priorities in forwarding the triggering message are determined by assigning different waiting times from the message reception to the moment at which they try to forward it. This waiting time is randomly computed within a contention window value, as inspired by classical backoff mechanisms in IEEE 802.11. However, in our scheme, the contention window value utilized by a vehicle is inversely proportional to the relative distance of the considered vehicle from the sender with respect to the ETR value.

If, while waiting, some other vehicle closer to the destination already forwarded the video triggering message, all vehicles between the sender and the current forwarder abort their countdowns to transmission as the message has already been propagated “over their heads”. Instead, all vehicles between the current forwarder and the destination will participate to the "forwarding contest" for the next hop. Obviously, the larger the contention window, the more likely somebody else will be faster in forwarding the video triggering message.

The contention window of each vehicle is measured in slots and varied between a minimum value (CWMin) and a maximum one (CWMax), depending on the distance from the sending/forwarding vehicle (Dist) and on the advertised ETR. This is summarized by (1).

$$
\left\lfloor\left(\frac{E T R-\text { Dist }}{E T R} \times(C W M a x-C W M i n)\right)+C W M i n\right\rfloor
$$

Clearly, this scheme ensures that the closest vehicle to the destination among those within the transmission range of the sender/forwarder will be statistically privileged in becoming the new forwarder.

\section{Experimental assessment}

We report results related to experiments where the length of the considered portion of road was varying from 4 to $20 \mathrm{Km}$. Considering a freeway with multiple lanes, several vehicle density conditions were generated as reported in Tab. 1 and vehicles' speeds were uniformly distributed in the range $72-144 \mathrm{Km} / \mathrm{h}$. To each of these settings we associated a plausible perhop delay as inspired by [9]. Taking inspiration from the real IEEE 802.11 protocol, we have set CWMin and CWMax equal to 32 and 1024 slots, respectively.

TABLE I. Traffic Densities (Multiple Lanes) Uniformly Distributed Cars

\begin{tabular}{|c|c|c|c|}
\hline $\begin{array}{c}\text { Traffic } \\
\text { Conditions }\end{array}$ & $\begin{array}{c}\text { Cars } \\
\text { per } \mathrm{Km}\end{array}$ & $\begin{array}{c}\text { Avg inter-vehicular } \\
\text { space }\end{array}$ & $\begin{array}{c}\text { Per-hop } \\
\text { delay }\end{array}$ \\
\hline very sparse & 60 & $17 \mathrm{~m}$ & $38 \mathrm{~ms}$ \\
\hline sparse & 90 & $11 \mathrm{~m}$ & $44 \mathrm{~ms}$ \\
\hline medium & 125 & $8 \mathrm{~m}$ & $66 \mathrm{~ms}$ \\
\hline dense & 190 & $5 \mathrm{~m}$ & $80 \mathrm{~ms}$ \\
\hline very dense & 240 & $4 \mathrm{~m}$ & $100 \mathrm{~ms}$ \\
\hline
\end{tabular}

We simulated different transmission ranges (i.e., 100, 300, 500, 700, and $1000 \mathrm{~m}$ ), yet, as a representative case and without any loss of generality, we present here only the results for $300 \mathrm{~m}$. For each vehicle density condition, 40 simulations were run and their outcomes averaged to produce charts presented in 
Section 6 (with 95\% confidence intervals).

We have compared Fast Triggering (FT in the following charts) with the following schemes that do not make use of dynamically estimated transmission range. Similar to our FT, Static is a scheme that assigns different forwarding priorities through random backoff delays that depend on the node distance from the source. However, this is done by assuming a predetermined maximum transmission range value, regardless of the actual one. Two instances of the Static scheme were employed: the Static scheme utilizing a fixed ETR value of $300 \mathrm{~m}$ (Static300) and the Static scheme utilizing a fixed ETR value of 1000m (Static1000). In essence, employing Static300 amounts to utilize (1) with a fixed ETR $=300$, and Static1000 corresponds to use (1) with ETR $=1000$. Clearly, Static scheme corresponds to the ideal case if and only if the actual transmission range matches the assumed one.

We also evaluated a scheme, RandomInc, that do not employ any distance prioritization in computing vehicles' contention window. More in detail, RandomInc employs a traditional backoff scheme where the contention window, comprised between CWMin and CWMax is doubled every time there is a collision, and reduced when a transmission is successfully performed, thus adapting to the congestion on the channel.

Results in Section 6 show that FT performs as well as the ideal Static algorithm. This result is achieved without requiring perfect knowledge of the network topology (just employing the transmission range estimator).

\section{Measured performance}

To compare the various schemes, we focused on parameters that have a direct delay impact, such as the total number of hops required to propagate the triggering message to the area-of-interest and the total number of messages sent.

In particular, Fig. 1 shows the average number of hops that a triggering message experiences to cover each $\mathrm{Km}$ of the road depending on traffic conditions (see Tab. 1); values in Fig. 1 represent averaged results with related confidence intervals. Clearly, FT performs with results comparable to those achieved by the ideal scheme where the actual transmission range is known a priori, i.e., Static300. This confirms that our scheme is able to adapt to the real channel conditions, finding out the actual transmission range and utilizing it to obtain the best performance, whereas other schemes require a higher number of hops to traverse each $\mathrm{Km}$. As the location of the next forwarder depends on the presence of priorities (if employed by the considered scheme) and not on the vehicle density, it is not surprising that the number of hops per $\mathrm{Km}$ is not influenced by the traffic conditions for none of the compared schemes.

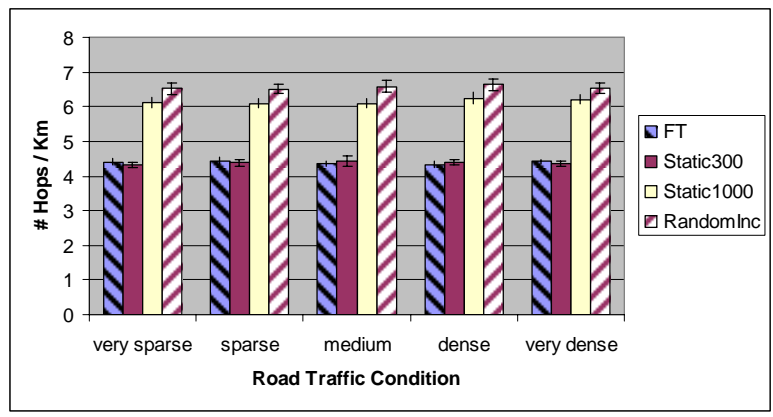

Figure 1. Avg no. of hops per Km.

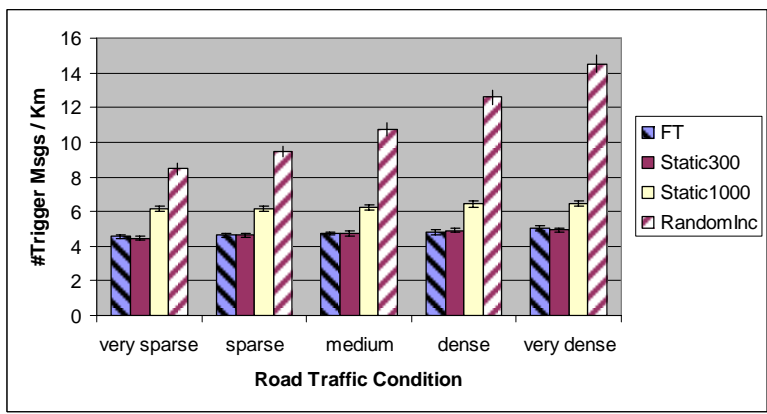

Figure 2. Avg no. of messages sent per Km.

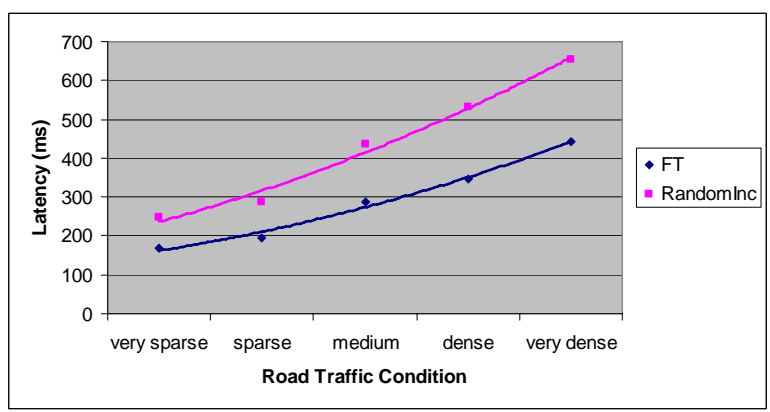

Figure 3. Delivery latencies of triggering messages per $\mathrm{Km}$.

To understand the causes of results in Fig. 1, we have to analyze the total number of messages sent per Km. To this aim, Fig. 2 shows that FT and the ideal scheme Static300 perform better than the others even considering this metric. This happens because both Static1000 and RandomInc do not employ an 
appropriate contention window. In particular, the former generally utilizes an inappropriately large contention window whereas, with the latter, vehicles generally use inappropriately small contention windows; in both cases, the final result is a reduced likelihood of farthest vehicles in becoming the next forwarders of the message. Moreover, as further causes of delays, large contention windows statistically generates longer waiting times before a message is sent and small contention windows statistically increase the number of packet collisions, especially with high vehicle density, thus requiring retransmissions to propagate the message.

Finally, to provide the reader with a tangible measure of the impact of the aforementioned number of hops on the final delivery time of a triggering message, we considered the per-hop latencies measured in [9] and multiplied their values (reported in Tab. 1) for the number of hops experienced with various vehicle densities (values in Fig. 1). In Fig. 3 we report outcomes of the best performer $(F T)$ and the worst one (RandomInc). As expected, FT has delivery latencies sensibly smaller than RandomInc. Moreover, even if the trend shows an increase of the transmission latency with the vehicle density for both schemes, with FT this phenomenon is more graceful than with RandomInc.

\section{Conclusion}

We considered the issue of broaden the view of first responders called to intervene in an crisis area. In particular, we tried to enable the remote and real time scrying of the emergency, while first responders are still driving toward the area. This crystal ball-like functionality would help first responders in proactively organize their actions, thus increasing the responsiveness and efficacy of their intervention.

In this context, one of the main problems is that of quickly triggering a live video (or any other kind of data, depending on the available devices) stream from a video camera located in the crisis area to an approaching first responders' vehicle. Addressing this problem, we discussed Fast Triggering: a scheme for multi-hop message delivery, able to bring triggering messages to destination with a reduced number of hops. As a main contribution, Fast Triggering is endowed with a novel transmission range estimator that permits efficient operations even in the highly variable inter-vehicular communications scenario.

\section{Acknowledgment}

Partial financial support for this work is provided by: the Italian MIUR (ICTP/E-Grid, MOMA, DAMASCO initiatives); the National Science Foundation (grants CNS-0435515/ANI-0221528); and STMicroelectronics (UC-CoRe Grant MICRO 05-06).

\section{References}

[1] America Rebuilds: A Year at Ground Zero, PBS. www.pbs.org/americarebuilds/engineering/engineering_qfact s.html

[2] Thousands of Security Webcams Wide Open. www.vnunet.com/vnunet/news/2126438/thousands-securitywebcams-wide-open

[3] ASTM E2213-03, "Standard Specification for Telecommunications and Information Exchange Between Road-side and Vehicle Systems - 5.9GHz Band Dedicated Short-Range Communications (DSRC) Medium Access Control (MAC) and Physical Layer (PHY) Specifications”, ASTM Inernational, Jul 2003.

[4] M. Guo, MH. Ammar, EW. Zegura, "V3: a Vehicle-toVehicle Live Video Streaming Architecture", Proc. 3rd IEEE International Conference on Pervasive Computing and Communications, Kauai, HI, Mar 2005.

[5] E. Fasolo, R. Furiato, A. Zanella, "Smart Broadcast Algorithm for Inter-vehicular Communication”, Proc. Wireless Personal Multimedia Communication (WPMC'05), Aalborg, DK, Sep 2005.

[6] G. Korkmax, E. Ekici, F. Ozguner, U. Ozguner, "Urban Multi-hop Bradcast Protocol for Inter-vehicle Communication Sustems", Proc. 1st ACM Workshop on Vehicular Ad-hoc Networks (VANET'04), Philadelphia, PA, Oct 2004.

[7] X. Yang, J. Liu, F. Zhao, N. Vaidya, “A Vehicle-toVehicle Communication Protocol for Cooperative Collision Warning”, Proc. 1st Annual International Conf. on Mobile and Ubiquitous Systems: Networking and Services (MobiQuitous'04), Boston, MA, Aug 2004.

[8] S. Biswas, R. Tatchikou, F. Dion, "Vehicle-to-Vehicle Wireless Communication Protocols for Enhancing Highway Traffic Safety”, IEEE Communication Magazine, 44(1):7482, Jan 2006.

[9] J. Yin, T. Elbatt, G. Yeung, B. Ryu, S. Habermas, H. Krishnan, T. Talty, "Performance Evaluation of Safety Applications over DSRC Vehicular Ad Hoc Networks", Proc. 1st ACM Workshop on Vehicular Ad-hoc Networks (VANET'04), Philadelphia, PA, Oct 2004. 